\title{
Analyst
}

CORRECTION

Cite this: Analyst, 2015, 140, 6421

\section{Correction: Cold shock induces apoptosis of dorsal root ganglion neurons plated on infrared windows}

\author{
DOI: $10.1039 / \mathrm{c5an} 90072 \mathrm{~g}$ \\ www.rsc.org/analyst \\ Ebrahim Aboualizadeh, ${ }^{a}$ Eric C. Mattson, ${ }^{a}$ Crystal L. O'Hara, ${ }^{\text {b }}$ Amanda K. Smith, ${ }^{\text {b }}$ \\ Cheryl L. Stucky ${ }^{b}$ and Carol J. Hirschmugl ${ }^{* a}$ \\ Correction for 'Cold shock induces apoptosis of dorsal root ganglion neurons plated on infrared windows' \\ by Ebrahim Aboualizadeh et al., Analyst, 2015, 140, 4046-4056.
}

In the Acknowledgements section, the NSF grant number is incorrect and should read 'CHE-1112433' instead of 'CHE-1114233'. The Royal Society of Chemistry apologises for these errors and any consequent inconvenience to authors and readers.

\footnotetext{
${ }^{a}$ Department of Physics, University of Wisconsin-Milwaukee, Milwaukee, WI 53211, USA. E-mail: cjhirsch@uwm.edu; Tel: +1 (414) 229-5748

${ }^{b}$ Department of Cell Biology, Neurobiology and Anatomy, Medical College of Wisconsin, Milwaukee, WI 53226-0509, USA
} 